\title{
Liver Transplantation because of Acute Liver Failure due to Heme Arginate Overdose in a Patient with Acute Intermittent Porphyria
}

\author{
Pascal Frei $^{\text {a }}$ Elisabeth I. Minder ${ }^{f} \quad$ Natascia Corti ${ }^{b}$ \\ Beat Muellhaupt $^{a} \quad$ Andreas Geier $^{a}$ Heiner Adams $^{d}$ \\ Jean-Paul Dutertre ${ }^{\mathrm{g}} \quad$ Alain Rudiger ${ }^{\mathrm{c}} \quad$ Philipp Dutkowski $^{\mathrm{e}}$ \\ Marco Maggiorini ${ }^{c}$ Christoph C. Ganter ${ }^{c}$ \\ ${ }^{a}$ Division of Gastroenterology and Hepatology, ${ }^{\mathrm{b}}$ Department of Pharmacology, \\ ${ }^{c}$ Medical Intensive Care Unit, ${ }^{d}$ Institute of Pathology and ${ }^{~}$ Department of \\ Surgery, University Hospital Zürich, and ${ }^{\mathrm{f}}$ Stadtspital Triemli, Zürich, \\ Switzerland; ${ }^{\text {OO}}$ Orphan Europe, Puteaux, France
}

\section{Key Words}

Heme arginate $\cdot$ Acute intermittent porphyria $\cdot$ Overdose $\cdot$ Acute liver failure $\cdot$ Liver transplantation

\begin{abstract}
In acute attacks of acute intermittent porphyria, the mainstay of treatment is glucose and heme arginate administration. We present the case of a 58-year-old patient with acute liver failure requiring urgent liver transplantation after erroneous 6 -fold overdose of heme arginate during an acute attack. As recommended in the product information, albumin and charcoal were administered and hemodiafiltration was started, which could not prevent acute liver failure, requiring super-urgent liver transplantation after 6 days. The explanted liver showed no preexisting liver cirrhosis, but signs of subacute liver injury and starting regeneration. The patient recovered within a short time. A literature review revealed four poorly documented cases of potential hepatic and/or renal toxicity of hematin or heme arginate. This is the first published case report of acute liver failure requiring super-urgent liver transplantation after accidental heme arginate overdose. The literature and recommendations in case of heme arginate overdose are summarized. Knowledge of a potentially fatal course is important for the management of future cases. If acute liver failure in case of heme arginate overdose is progressive, super-urgent liver transplantation has to be evaluated.
\end{abstract}




\section{Introduction}

Acute intermittent porphyria (AIP) is an autosomal dominant disorder of the heme biosynthetic pathway [1]. Symptoms of AIP manifest as acute crises and are frequently precipitated by environmental factors such as the ingestion of porphyrinogenic drugs, alcohol overdose, fasting or stress. Patients with an acute attack of AIP present with neurovisceral symptoms such as abdominal pain, nausea, vomiting, sensory and motor neuropathy, and neuropsychiatric symptoms. Treatment is supportive, with avoidance of precipitating factors and administration of carbohydrates and hemin (heme arginate or hematin). Hemin represses hepatic delta-aminolevulinic acid synthase, the rate-limiting enzyme in heme biosynthesis. Compared to hemin, heme arginate is a more stable preparation and does not cause coagulopathy or phlebitis [2]. We describe a case of acute liver failure due to accidental 6-fold overdose of heme arginate requiring liver transplantation.

\section{Case Report}

A 58-year-old woman weighing $51 \mathrm{~kg}$ with AIP since 35 years was hospitalized in a secondary care hospital due to an acute attack of AIP. Past medical history revealed arterial hypertension, chronic renal insufficiency (creatinine $298 \mu \mathrm{mol} / \mathrm{l}$ ) with secondary hyperparathyroidism and chronic neuropathy, which were all associated with AIP and were being treated with propranolol $40 \mathrm{mg} /$ day and 1,25-dihydroxycholecalciferol. Initially, the AIP crisis was treated with intravenous glucose, following which symptoms improved and urinary porphobilinogen excretion decreased. Two weeks later, AIP recurred and heme arginate was administered. One hour after the heme arginate infusion, it became apparent that an accidental 6-fold overdose of heme arginate had been given - 1,000 mg (19 $\mathrm{mg} / \mathrm{kg}$ body weight instead of the recommended daily dose of $3 \mathrm{mg} / \mathrm{kg}$ body weight).

The patient was transferred to the intensive care unit. As recommended in the product information, intravenous infusions of glucose $20 \%$ and of albumin ( 20 g repeatedly) were given to bind free hemin, and repetitive oral charcoal suspensions (a total of $90 \mathrm{~g}$ from $3.5 \mathrm{~h}$ to $48 \mathrm{~h}$ after overdose) were administered to interrupt the enterohepatic recirculation of hemin. Nevertheless, the patient developed acute liver failure, with rapidly rising transaminases and worsening coagulation (fig. 1). With substitution of glucose $50 \%$, no hypoglycemia was apparent. The patient became oliguric, and continuous venovenous hemodiafiltration (CVVHDF) was started. The patient remained hemodynamically stable. Viral hepatitis was excluded. During the 2 days before heme arginate overdose, the patient had received daily doses of 10,000 $\mathrm{U}$ of subcutaneous heparin, $60 \mathrm{mg}$ propranolol, 2,400 mg sevelamer, $600 \mathrm{mg}$ aspirin, $3.75 \mathrm{mg}$ midazolam, $15 \mathrm{mg}$ oxazepam and $0-2 \mathrm{~g}$ paracetamol, with a maximum dose of paracetamol of 3 g given on the day heme arginate was initiated. Thereafter, paracetamol was discontinued, with no paracetamol detectable in the serum $25 \mathrm{~h}$ after the overdose. Treatment with sevelamer and propranolol was continued and the patient was given $10 \mathrm{mg}$ metoclopramide, macrogol, lactulose, $1 \mathrm{~g}$ metamizole and 10-12 mg morphine daily and a single dose of $10 \mathrm{mg}$ of vitamin K. No coagulation products had to be given. After $48 \mathrm{~h}$, the patient was transferred to our tertiary care hospital due to deteriorating acute liver failure and listed for super-urgent liver transplantation. Coagulation was worsening (coagulation factor $\mathrm{V}$ unmeasurable), CVVHDF was being continued due to anuric renal failure, and the patient developed encephalopathy grade I-II. $62 \mathrm{~h}$ after the overdose, transaminases peaked and coagulation improved (coagulation factor V 12\%), suggesting spontaneous liver recovery. However, on the 5 th day, liver function again deteriorated, with worsening coagulation and deteriorating encephalopathy necessitating intubation and mechanical ventilation. After 6 days, a liver graft from a deceased donor was available and the patient was transplanted. Histo pathological analysis of the liver explant revealed subacute toxic liver injury without signs of underlying liver disease (fig. 2). One year later, the patient is in good condition and the AIP seems to have been cured by the liver transplantation, although she is still on intermittent hemodialysis. 


\section{Discussion}

Data on the liver toxicity of hematin or heme arginate overdoses are scarce. In mice and rats, the lowest lethal dose was at least 10 -fold greater than the recommended therapeutic dose (3 mg/kg i.v.) for humans. LD50 in mice was $56.3 \mathrm{mg} / \mathrm{kg}$ heme arginate. Mice died within the first days [3], and an infarcted liver with necrosis of varying extensions was observed [4]. In humans, no data on the liver toxicity of hemin are available in the recent literature or common databases such as PubMed or DRUGDEX (Ref: DRUGDEX ${ }^{\circledR}$ System, Thomson Reuters, Greenwood Village, Colorado, edition 2009). Only 1 case of liver enzyme elevation was found in the International Adverse Event Reporting System of the World Health Organization after giving intravenous heme arginate of unknown dosage. However, to elucidate the present case, an intensive search revealed 4 poorly documented case reports of potential hepatic and/or renal toxicity of hematin or heme arginate. The first report described a 40-year-old patient with AIP who developed transient renal failure after rapid administration of $1,000 \mathrm{mg}$ of hematin [5]. In a second case, a patient received $3,000 \mathrm{mg}$ of heme arginate over 2 days. He developed acute liver failure with hyperbilirubinemia, anemia and generalized hemorrhagic diathesis lasting for several days, but then improved spontaneously [6]. In the third case, a patient received a 10 -fold higher dose than recommended as a single infusion as preoperative prophylaxis. He developed hyperbilirubinemia $(114 \mu \mathrm{mol} / \mathrm{l})$ and a marked rise in aspartate aminotransferase (1,545 U/l), without clinically overt bleeding [7]. In the last case, a patient accidentally received 2,500 $\mathrm{mg}$ of heme arginate as a single infusion and developed acute liver failure, requiring urgent liver transplantation. After a complicated postoperative course requiring a second transplantation, the patient died (Ulrich Stölzel, Chemnitz, Germany; personal communication in April 2010). No further information is available on this case.

In our patient, the 6-fold overdose of heme arginate is the probable cause of her acute liver failure. There are several reasons in support of this. First, no other causative toxin could be identified. Even though paracetamol had been administered, the maximum daily dose was $3 \mathrm{~g} /$ day, and no paracetamol was detectable in the serum $25 \mathrm{~h}$ after its last administration. The second potentially hepatotoxic substance was propylene glycol, which is the solvent of heme arginate. However, 1,000 mg heme arginate are solved in only $16 \mathrm{~g}$ of propylene glycol, and the maximum daily dose of propylene glycol considered to be safe is more than $69 \mathrm{~g} /$ day [8]. Hence, the dose of $16 \mathrm{~g}$ of propylene glycol given seems insignificant, particularly in view of a normal anion gap in the blood. Second, viral hepatitis, hypoperfusion of the liver or a vascular cause for acute liver failure could be excluded. Third, histological examination of the explanted liver showed typical changes of toxic liver injury with no evidence of preexisting liver disease. Fourth, the time course of acute liver failure is typical of drug-induced acute liver failure.

One can speculate about the potential mechanism of heme arginate liver toxicity. Heme arginate, the arginate salt of ferric protoporphyrin, is primarily bound to albumin and taken up by hepatocytes. Heme arginate overdose may lead to accumulation of free hemin in the blood circulation, which may cause significant toxicity in different organs [9]. In the liver, free heme might damage mitochondrial DNA and induce apoptosis. After several therapeutic heme arginate infusions, no significant accumulation of iron 
or heme was found [10]. In our case of heme arginate overdose, no hepatic iron overload was detectable by iron staining of the liver explant 6 days after exposure, but early accumulation of heme after the overdose might be expected.

In summary, heme arginate is the treatment of choice for acute attacks of AIP. However, heme arginate overdose may lead to acute liver failure. The suggested management of heme arginate overdose includes repeated oral charcoal suspension administration to interrupt the enterohepatic recirculation of heme, albumin infusions to bind free and toxic heme, and hemodiafiltration to eliminate hepatotoxic and nephrotoxic propylene glycol. If this therapy fails and acute liver failure is progressive, super-urgent liver transplantation has to be evaluated. In AIP, the excess production of porphyrin intermediates occurs exclusively in the liver.

Liver transplantation cures the patient from acute porphyria. Liver transplantation has so far only been performed in individual cases with an otherwise untreatable course of disease, including frequently recurring life-threatening acute attacks leading to extremely poor quality of life [11-15].

\section{Acknowledgement}

The authors thank Dr. Ewerton Marques Maggio for his help in the preparation of fig. 2. 

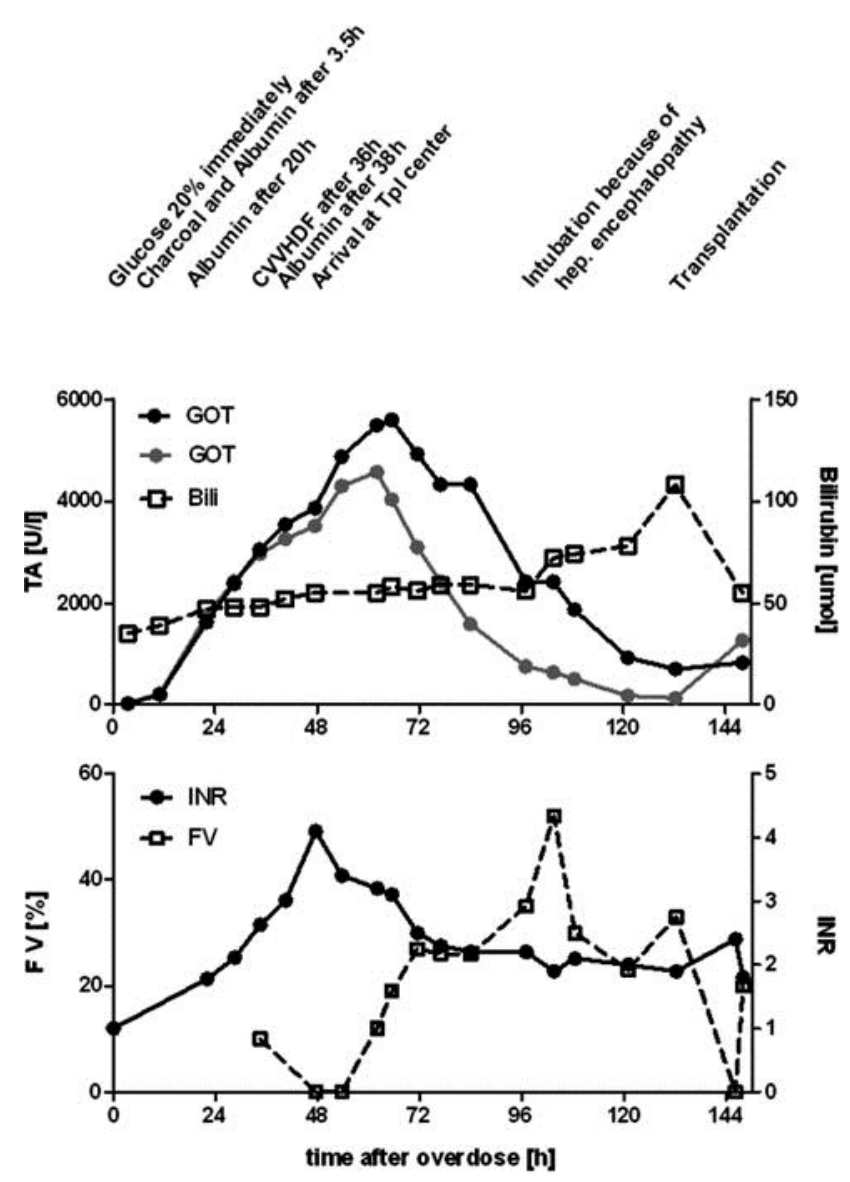

Fig. 1. Time course of blood tests: aspartate aminotransferase (U/l), alanine aminotransferase (U/l), bilirubin $(\mu \mathrm{mol} / \mathrm{l})$, lactate dehydrogenase $(\mathrm{U} / \mathrm{l})$, coagulation factor $\mathrm{V}(\%)$, international normalized ration (INR), lactate (mmol/l) and ammonia ( $\mu \mathrm{mol} / \mathrm{l})$. 

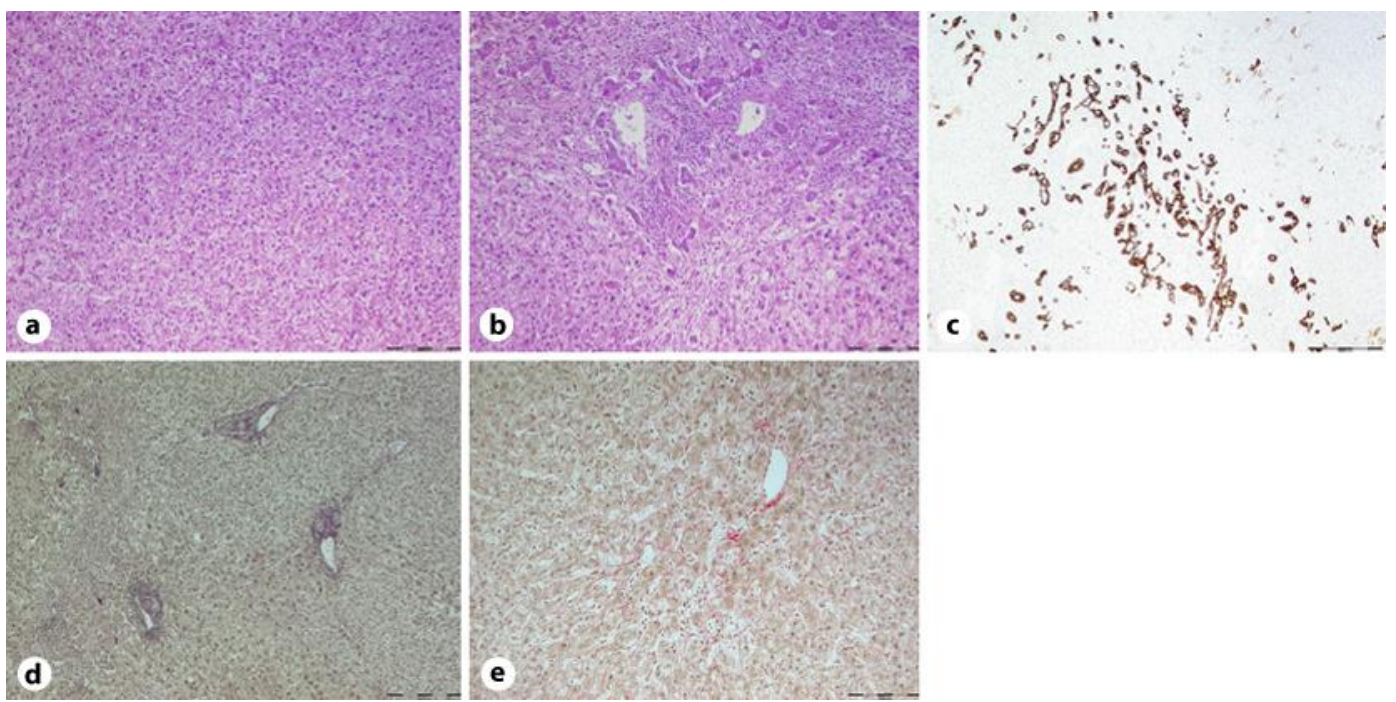

Fig. 2. Histopathological examination of the liver explant was suggestive of subacute toxic liver injury with little signs of histological recovery and without signs of underlying chronic liver disease such as fibrosis or cirrhosis. a Hypoplastic and hyperplastic hepatocytes. b Resorptive inflammation and ductular proliferation. c Ductular proliferates as a sign of regeneration (cytokeratin 7 immunostaining). $\mathbf{d}$ No preexisting fibrous septa or cirrhosis (Elastica van Gieson staining). e Sinusoidal fibrous septa seen as a sign of reparation (Sirius staining). Scale bars represent $200 \mu \mathrm{m}$ in panels a-d and $100 \mu \mathrm{m}$ in panel e.

\section{References}

1 Anderson KE, Bloomer JR, Bonkovsky HL, Kushner JP, Pierach CA, Pimstone NR, Desnick RJ: Recommendations for the diagnosis and treatment of the acute porphyrias. Ann Intern Med 2005;142:439-450.

2 Volin L, Rasi V, Vahtera E, Tenhunen R: Heme arginate: effects on hemostasis. Blood 1988;71:625-628.

3 Normosang product information. Orophan Europe. http://www.medicines.org.uk/emc/medicine/ 20795/SPC/Normosang/.

4 Tenhunen R, Tokola 0, Linden IB: Haem arginate: a new stable haem compound. J Pharm Pharmacol 1987;39:780-786.

5 Dhar GJ, Bossenmaier I, Cardinal R, Petryka ZJ, Watson CJ: Transitory renal failure following rapid administration of a relatively large amount of hematin in a patient with acute intermittent porphyria in clinical remission. Acta Med Scand 1978;203:437-443.

-6 Kostrzewska E, Gregor A, Tarczynska-Nosal S: Heme arginate (Normosang) in the treatment of attacks of acute hepatic porphyrias. Mater Med Pol 1991;23:259-262.

7 Orphan Europe, internal data.

-8 Zar T, Graeber C, Perazella MA: Recognition, treatment, and prevention of propylene glycol toxicity. Semin Dial 2007;20:217-219.

\$9 Kumar S, Bandyopadhyay U: Free heme toxicity and its detoxification systems in human. Toxicol Lett 2005;157:175-188.

$\checkmark 10$ Ventura P, Cappellini MD, Rocchi E: The acute porphyrias: a diagnostic and therapeutic challenge in internal and emergency medicine. Intern Emerg Med 2009;4:297-308.

11 Dar FS, Asai K, Haque AR, Cherian T, Rela M, Heaton N: Liver transplantation for acute intermittent porphyria: a viable treatment? Hepatobiliary Pancreat Dis Int 2010;9:93-96.

12 Seth AK, Badminton MN, Mirza D, Russell S, Elias E: Liver transplantation for porphyria: who, when, and how? Liver Transpl 2007;13:1219-1227. 
13 Soonawalla ZF, Orug T, Badminton MN, Elder GH, Rhodes JM, Bramhall SR, Elias E: Liver transplantation as a cure for acute intermittent porphyria. Lancet 2004;363:705-706.

14 Stojeba N, Meyer C, Jeanpierre C, Perrot F, Hirth C, Pottecher T, Deybach JC: Recovery from a variegate porphyria by a liver transplantation. Liver Transpl 2004;10:935-938.

15 Wahlin S, Harper P, Sardh E, Andersson C, Andersson DE, Ericzon BG: Combined liver and kidney transplantation in acute intermittent porphyria. Transpl Int 2010;23:e18-e21. 